\title{
Adaptaciones curriculares para mejorar el rendimiento académico en niños con NEE de Educación Básica
}

\section{Curricular adaptations to improve academic performance in children with SEN in elementary school}

DOI: $10.46932 / \mathrm{sfjdv} 2 \mathrm{n} 5-131$

Received in: Oct 1st, 2021

Accepted in: Dec 30th, 2021

Lucia Mirella Sanisaca Guzmán

Aspirante a Doctora en Educación de la Universidad César Vallejo, Piura-Perú

Magister en Diseño Curricular. Directora de la Esc

León de Febres Cordero, Guayaquil, Guayas, Ecuador

Ciudadela Simón Bolívar calle 14A N-E y $4^{\circ}$ Pasaje 2A N-E

Correo electrónico: lucia.sanisaca@educacion.gob.ec

Mario Napoleón Briones Mendoza

Docente de la Universidad César Vallejo, Piura-Perú

Doctor en Educación

Director de la Institución Educativa Libertadores de América, La Unión, Piura, Perú

Av. Chulucanas s/n, Piura 20001, Perú

Correo electrónico: mbrionesm@ucvvirtual.edu.pe

\section{Elvira Armenia Játiva Sarcos}

Aspirante a Doctora en Educación de la Universidad César Vallejo, Piura-Perú

Magister en Diseño Curricular

Directora de la Esc

Pedro Vicente Maldonado, Guayaquil, Guayas, Ecuador

Aspirante a Doctora en Educación de la UCV

Coronel 904 Maldonado

Correo electrónico: elvira.jativa@educacion.gob.ec

\section{Gina Elizabeth Mera Moya}

Aspirante a Doctora en Educación de la Universidad César Vallejo, Piura-Perú

Máster Universitario en Formación Internacional Especializada del profesorado por la Universidad

Complutense de Madrid

Docente de Lengua y Literatura de la Unidad Educativa "Sarah Flor Jiménez

Cdla Colinas del maestro mz 640 villa 29 Guayaquil-Ecuador

1-4 Escuela de Posgrado, programa académico doctorado en educación, Universidad César Vallejo, Piura-Perú.

Correo electrónico: gina.mera@educacion.gob.ec

\section{RESUMEN}

La investigación tuvo como propósito principal proponer una guía de adaptaciones curriculares para mejorar el rendimiento académico de los estudiantes con NEE del Distrito 09D05C04, a partir de la caracterización, identificación y descripción de las diferentes estrategias específicas y diversificadas ya 
sea para la discapacidad permanente o transitoria. Este estudio se realizó con 81 educadores con el enfoque cuantitativo, diseño proyectivo, con una muestra no probabilística. La técnica empleada para la recolección de la información fue la observación sistematizada con el instrumento lista de cotejo; la guía de observación, estuvo compuesto de 24 ítems donde describía si tiene o no tienen los documentos legales. El instrumento no requiere de validación de expertos, puesto que la comprensión del mismo es para la autora. Los resultados fueron analizados e interpretados por el programa SPSS versión 2.5 para luego distribuirlos en tablas y figuras respectivamente, encontrando que aproximadamente un $93 \%$ de docentes (Tablas del 1 al 9) no cuentan con los documentos legales para la elaboración y uso de las adaptaciones curriculares, llegando a identificar la necesidad de la elaboración de una propuesta con estrategias específicas y diversificadas, adicionalmente, incluir en la planificación el factor del entorno familia y la evaluación cuantitativa, para proporcionar la guía a los docentes del Distrito 09D05C04. Estos resultados llevaron a concluir que los docentes necesitan de un medio (Guía) que les permita hacer adaptaciones curriculares para mejorar el rendimiento académico en niños con NEE de Educación Básica.

Palabras claves: Adaptaciones curriculares, estrategias específicas, estrategias diversificadas, evaluación cuantitativa, rendimiento académico.

\begin{abstract}
The main purpose of the research was to propose a guide for curricular adaptations to improve the academic performance of students with SEN in District 09D05C04, based on the characterization, identification and description of the different specific and diversified strategies for either permanent or temporary disability. This study was carried out with 81 educators with the quantitative approach, projective design, with a non-probabilistic sample. The technique used to collect the information was systematic observation with the checklist instrument; The observation guide was made up of 24 items describing whether or not they have the legal documents. The instrument does not require validation by experts, since its understanding is for the author. The results were analyzed and interpreted by the SPSS version 2.5 program and then distributed in tables and figures respectively, finding that approximately 93\% of teachers (Tables 1 to 9) do not have the legal documents for the preparation and use of the adaptations. curricular, coming to identify the need for the elaboration of a proposal with specific and diversified strategies, additionally, to include in the planning the factor of the family environment and the quantitative evaluation, to provide guidance to the teachers of District 09D05C04. These results led to the conclusion that teachers need a medium (Guide) that allows them to make curricular adaptations to improve academic performance in children with SEN in Basic Education.
\end{abstract}

Keywords: curricular adaptations, specific strategies, diversified strategies, quantitative assessment, academic performance.

\title{
1 INTRODUCCIÓN
}

El rendimiento académico siempre ha sido evaluado mediante pruebas estandarizadas, por ejemplo, las Pruebas Pisa donde sitúan en una escala de mayor a menor el nivel de enseñanza de los escolares, en otras palabras, el país que obtuvo mejores calificaciones es el que tiene la mejor educación. Pero no están demostrando una verdadera equidad, porque se centran en evaluar habilidades, competencias y destrezas de los estudiantes en las áreas principales sin considerar la diversidad de estudiantes que se tiene en cada país. ¿Pero qué sucede con aquellos escolares que tienen NEE? Los informes de dichas pruebas no han considerado las NEE de los niños y adolescentes, las pruebas son 
tomadas en base a los estándares de calidad internacional y no tienen igualdad de oportunidades para todos como lo establece el objetivo sostenible de la UNESCO.

De manera semejante, LLECE a nivel latinoamericano está centrada en evaluar y medir los logros de aprendizajes alcanzados de los niños de tercero y sexto grado en el área de matemática, lengua y ciencias de países como: Costa Rica, Perú, Nicaragua, El Salvador, Ecuador, entre otros. Todavía no se han publicado los resultados evaluados en el 2019, pero no se evidencia pruebas destinadas para establecer el rendimiento académico de los educandos con NEE, a pesar, de que mencionan que este tipo de mediciones permiten cumplir con el cuarto objetivo sostenible.

Los resultados de la Prueba Pisa Ecuador tienen las mismas características debido a que son estandarizadas, donde los niveles de logro de los aprendizajes están por el inferior de la puntuación a nivel latinoamericano, tanto en lectura, matemática y ciencias, de todo esto se desprende que, el rendimiento académico no cumple con los estándares internacionales. A pesar que dentro del informe determinan la equidad en el desempeño en las pruebas, esto no se evidencia, debido a que los estudiantes tienen sus respectivos carnets de discapacidad, diagnósticos con NEE no asociadas a la discapacidad, fueron evaluados de la misma manera curricular que el resto de los estudiantes, sin adaptar las evaluaciones.

En el Distrito 09D05 de la Zona 8 de Guayaquil, se entregó de manera aleatoria los kits donde se encontraban las pruebas que se tomarían en el año 2019 a los educandos de cuarto y séptimo grado de los diferentes planteles de la localidad, en igual forma, no solicitaron los respectivos listados de estudiantes que tienen NEE, vulnerando la igualdad para los estudiantes que requieren una adaptación para las pruebas estandarizadas, estos procesos no han sido contemplados desde las políticas nacionales e internacionales.

Las diferentes escuelas son orientadas por asesores educativos donde socializan los documentos entregados del Ministerio de Educación Ecuador para conocer cómo está el rendimiento académico de los escolares NEE, un instructivo donde explican todos los niveles de logros que requieren los estudiantes, después de tener una valoración o carnet de discapacidad que determine su necesidad educativa y cuáles deben ser los criterios de evaluación fomentando la participación en clase.

Los establecimientos educativos del Distrito 09D05C4 deben impulsar acciones que permitan elevar del rendimiento académico de los estudiantes con NEE, con diferentes adaptaciones curriculares según las necesidades educativas que presente el estudiante, por ejemplo, si tiene discapacidad auditiva, motriz, síndrome de Down, asperger, hiperactividad, entre otras que permitan obtener una equidad a la educación, con diferentes destrezas según el grado de dificultad que tenga.

Los estudiantes con NEE con o sin discapacidad requieren de diferentes adecuaciones en el currículo nacional, y los docentes pertenecientes al Distrito 09D05C04 no se encuentra preparados para realizar las respectivas evaluaciones con diferenciación de aprendizajes, de tal manera, que las 
calificaciones no son el resultado de una planificación adecuada, basada con las metodologías inclusivas respetando la diversidad y equidad.

¿Qué estrategias de planificación debe contar una guía de adaptaciones curriculares para mejorar el rendimiento académico en niños con NEE de Educación Básica de la ciudad de Guayaquil, 2021? Adicionalmente los problemas específicos que son: ¿Qué características tienen las estrategias diversificadas y específicas que utilizan los docentes para el rendimiento académico de los estudiantes con NEE?,¿Qué elementos debe tener una guía de adaptaciones curriculares para docentes, que permita mejorar el rendimiento académico en los niños con NEE de Educación Básica de la ciudad de Guayaquil?, ¿Cuáles son las características que tienen las estrategias diversificadas y específicas que utilizan los docentes para el rendimiento académico de los estudiantes con NEE? Y ¿Cómo elaborar una guía de adaptaciones curriculares para docentes, que permita mejorar el rendimiento académico en los niños con NEE de Educación Básica de la ciudad de Guayaquil?

Ante esta situación, realizar el presente estudio es conveniente porque permite orientar a los educadores con estrategias específicas y diversificadas en el contexto educativo, aplicando en los diferentes documentos legales, por ejemplo, el Documento Individual de Adaptación Curricular, guía y manual de adaptaciones para que se planifiquen adecuadamente los instrumentos y criterios de evaluación según el grado 1,2 o 3 de dificultades de aprendizaje.

En la actualidad permitirá obtener un valor teórico, porque se logrará llenar el conocimiento con las estrategias específicas y diversificadas que se deben emplear con los estudiantes con NEE, obteniendo buenos resultados con las adaptaciones curriculares apoyándose con la teoría del constructivismo debido a que los docentes del Distrito 09D05C04 no elaboran de manera adecuada las adaptaciones de los niños que estudian en los diferentes planteles educativos. Adaptar el currículo para los niños con NEE es uno de los objetivos de desarrollo sostenible que impulsa la calidad de la educación.

Como objetivo general se plantea el siguiente: Proponer una guía de adaptaciones curriculares de NEE para mejorar el rendimiento académico en niños de Educación Básica de la ciudad de Guayaquil, 2021.

Los objetivos específicos son: Caracterizar las adaptaciones curriculares de estudiantes con NEE que realizan los docentes de las aulas inclusivas. Identificar los elementos que debe tener una guía de adaptaciones curriculares para docentes, que permita mejorar el rendimiento académico en los niños con NEE de Educación Básica de la ciudad de Guayaquil. Describir las características que tienen las estrategias diversificadas y específicas que utilizan los docentes para el rendimiento académico de los estudiantes con NEE. Identificar los elementos que aportan a la propuesta para reducir las causas del rendimiento académico en niños con NEE de Educación Básica Circuito 09D05C04 de Guayaquil, 2021. 


\section{MARCO TEÓRICO}

En lo que respecta a la variable de rendimiento académico en los estudiantes con necesidades educativas espaciales a nivel internacional, Sabando (2016) realizó una tesis cuyo título es "Inclusión educativa y rendimiento académico" con el fin de expresar la importancia de la integración de los estudiantes en el régimen escolar habitual. El estudio se realizó con una metodología cuantitativa no experimental, de carácter correlacional y descriptivo; donde se toma una muestra de manera aleatoria, con el propósito de analizar la inclusión de los centros educativos y el rendimiento académico que tienen los escolares, correlacionando el efecto que tiene una de la otra. Dentro de las conclusiones, determina que es necesario emplear la heterogeneidad para agrupar a los estudiantes, con la finalidad de potenciar las individualidades y disminuir las dificultades de aprendizaje.

A continuación, se presenta los estudios relacionados con la variable de adaptaciones curriculares, en lo cual se describe la investigación de Chávez (2016) titulado Adaptaciones curriculares e inclusión escolar con discapacidad intelectual a partir de la percepción de los docentes de primaria, Ica - 2016 con el enfoque cuantitativo, con diseño descriptivo correlacional, no experimental considerando específicamente con 54 educadores empleado un cuestionario con la prueba Tao b de Kendall. Los resultados han demostrado que si es necesario utilizar las diferentes actividades que permitan realizar en el ciclo de aprendizaje las actividades respectivas debido a que las correlaciones de las variables han permitido determinar la importancia del mismo.

\section{METODOLOGÍA}

El trabajo investigativo se centró en el enfoque cuantitativo con el método analítico que permite descomponer la variable teórica: adaptaciones curriculares y variable fáctica: rendimiento académico en niños con NEE porque fue necesario analizar la realidad del contexto educativo frente a la problemática inclusiva. En vista que no todos los educadores realizan adecuaciones de acceso al currículo de manera óptima y es necesario que genere nuevos conocimientos de esta temática.

Además, es de tipo no experimental donde se realizará un diagnóstico que presenta la problemática con la finalidad de diseñar adaptaciones curriculares para mejorar el rendimiento académico en niños con NEE de Educación Básica de doce planteles educativos de la ciudad de Guayaquil, 2021. Según el propósito se considera de manera descriptiva porque se analizará el rendimiento que tienen los estudiantes en las asignaturas de matemática, ciencias lengua y literatura para realizar la propuesta antes mencionada.

Además, es propositiva. De tal manera, que las variables fueron medidas con los instrumentos adecuados para mejorar en el contexto educativo. Según su periodo de tiempo es de carácter transversal debido a que se realizará un diagnóstico por una sola vez para determinar si los docentes han elaborados los Documentos Curriculares a los estudiantes con NEE. El diagnóstico fue por medio de la observación 
de los documentos de adaptaciones curriculares que tienen los educadores, las respectivas planificaciones y evaluaciones del periodo lectivo 2021-2022.

Tabla 1. Población

\begin{tabular}{ll}
\hline Cargo & Cantidad \\
\hline Docentes de nombramiento & 58 \\
Docentes contratado & 23 \\
Total & 81 \\
\hline
\end{tabular}

Fuente: Distrito 09D05C04

La población descrita en la Tabla ${ }^{\circ} 1$ se detallan a 81 docentes de los planteles educativos de la ciudad de Guayaquil del Distrito 09D05C04. La muestra es un subgrupo de personas que están seleccionadas de manera aleatoria que comprende la totalidad de la población denominándola como muestra censal (Hernández y otros, 2014). Del mismo sentido, el criterio de clasificación de la muestra en esta investigación se estimó el muestreo con carácter no probabilístico debido a que no todos los educadores están concentrados en un solo establecimiento educativo, pero si de un mismo Distrito excluyendo aquellos que no tienen estudiantes con NEE.

\section{RESULTADOS}

\subsection{DESCRIPCIÓN DE RESULTADOS}

OE1: Caracterizar las adaptaciones curriculares de estudiantes con NEE que realizan los docentes de las aulas inclusivas.

Tabla 2 Datos de la dimensión factores del rendimiento académico: Factor del entorno familiar.

\begin{tabular}{|c|c|c|c|c|c|c|}
\hline \multirow[b]{2}{*}{ Factor del entorno familiar } & \multicolumn{2}{|l|}{$\mathrm{NO}$} & \multicolumn{2}{|l|}{ SI } & \multicolumn{2}{|c|}{ Total } \\
\hline & $\mathrm{N}^{\circ}$ & $\%$ & $\mathrm{~N}^{\circ}$ & $\%$ & $\mathrm{~N}^{\circ}$ & $\%$ \\
\hline $\begin{array}{l}\text { 1. En el DIAC, relata el entorno } \\
\text { familiar en la que tiene el estudiante }\end{array}$ & 47 & $58 \%$ & 34 & $42 \%$ & 81 & $100 \%$ \\
\hline $\begin{array}{l}\text { 2. En el DIAC, cuenta con } \\
\text { detalles familiares relacionada a su } \\
\text { discapacidad o problema familiar }\end{array}$ & 44 & $54 \%$ & 37 & $46 \%$ & 81 & $100 \%$ \\
\hline $\begin{array}{l}\text { 3. En el DIAC, detalla las } \\
\text { características de la familia en la } \\
\text { educación del estudiante }\end{array}$ & 61 & $75 \%$ & 20 & $25 \%$ & 81 & $100 \%$ \\
\hline
\end{tabular}

Fuente: Lista de cotejo aplicado a los docentes del Educación Básica Circuito 09D05C04 de Guayaquil. 
Figura 1 Datos Niveles y porcentajes del indicador factor del entorno familiar

\section{Factor del entorno familiar}

3. En el Documento Individual de Adaptación Curricular, detalla las características de la familia en la educación del estudiante

2. En el Documento Individual de Adaptación Curricular, cuenta con detalles familiares relacionada a su discapacidad o problema familiar

1. En el Documento Individual de Adaptación Curricular, relata el entorno familiar en la que tiene el estudiante

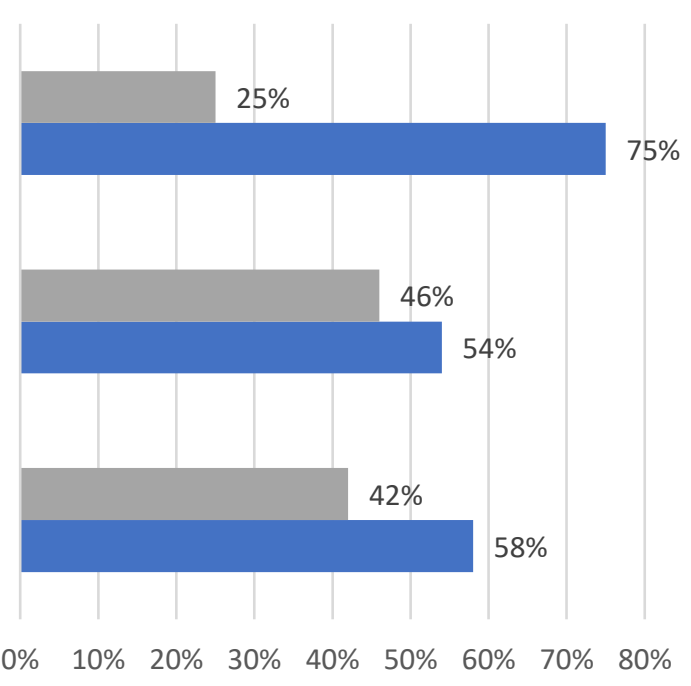

Fuente: Lista de cotejo aplicado a los docentes del Educación Básica Circuito 09D05C04 de Guayaquil.

En los datos obtenidos de la dimensión factores del rendimiento académico en la tabla 1 se observó a los docentes, el 58\% no contaban con el relato del entorno familiar de los estudiantes con NEE en el documento individual de Adaptación Curricular, de igual manera el 54\% tampoco contó con los detalles familiares relacionados con la discapacidad o problema que ha causado el problema de aprendizaje, adicionalmente el 75\% no detalló las características de los familiares en relación al compromiso que tuvieron con los estudiantes en la educación antes de ese periodo lectivo. Esto quiere decir, que no se evidencia la responsabilidad de los familiares en cuanto al conocimiento que requieren los estudiantes para aprender las destrezas con criterio de desempeño y sobre todo la responsabilidad que deben asumir los educadores para integrar a las familias a la inclusión educativa del infante.

Tabla 3 Datos de la dimensión factores del rendimiento académico: Factor del entorno social.

\begin{tabular}{|c|c|c|c|c|c|c|}
\hline \multirow{2}{*}{ Factor del entorno social } & \multicolumn{2}{|l|}{$\mathrm{NO}$} & \multicolumn{2}{|l|}{ SI } & \multicolumn{2}{|c|}{ Total } \\
\hline & $\mathrm{N}^{\circ}$ & $\%$ & $\mathrm{~N}^{\circ}$ & $\%$ & $\mathrm{~N}^{\circ}$ & $\%$ \\
\hline $\begin{array}{l}4 . \quad \text { En el DIAC, cuenta con } \\
\text { adaptaciones de las habilidades } \\
\text { sociales }\end{array}$ & 54 & $67 \%$ & 27 & $33 \%$ & 81 & $100 \%$ \\
\hline $\begin{array}{l}5 . \quad \text { En el DIAC, plantea } \\
\text { actividades interactivas con el resto } \\
\text { de estudiantes. }\end{array}$ & 57 & $70 \%$ & 24 & $30 \%$ & 81 & $100 \%$ \\
\hline $\begin{array}{l}\text { 6. En el DIAC, elabora } \\
\text { intervención } \\
\text { especialistas internos y externos. }\end{array}$ & 48 & $59 \%$ & 33 & $41 \%$ & 81 & $100 \%$ \\
\hline
\end{tabular}


Figura 2 Niveles y porcentajes del indicador factor del entorno social

\section{Factor del entorno social}

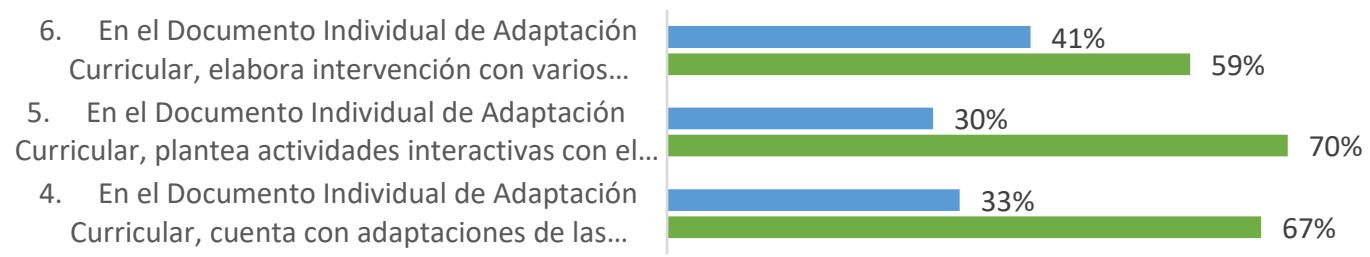

Fuente: Lista de cotejo aplicado a los docentes del Educación Básica Circuito 09D05C04 de Guayaquil.

Los resultados obtenidos en la dimensión de factores del rendimiento académico, factor del entorno social, el 67\% de los docentes no contó con adaptaciones de las habilidades sociales dentro del DIAC (Documento Individual de Adaptación Curricular), del mismo modo el $70 \%$ no describió actividades interactivas con el resto de estudiantes; adicionalmente el 59\% tampoco elaboró la descripción de la intervención con varios especialistas internos y externos que requieren los estudiantes con NEE. Asimismo, más del $50 \%$ de la observación que se realizó, el DIAC no cuenta con intervención de especialistas internos y externos, no plantean actividades interactivas y adaptaciones de las habilidades sociales. Las adaptaciones de las habilidades sociales con especialistas internos y externos permiten planificar actividades con estrategias específicas para la discapacidad que tiene el estudiante.

Tabla 4 Datos de la dimensión factores del rendimiento académico: Factor del entorno escolar.

\begin{tabular}{lllllll}
\hline \multirow{2}{*}{ Factor del entorno escolar } & $\mathrm{NO}$ & \multicolumn{3}{c}{$\mathrm{SI}$} & \multicolumn{2}{c}{ Total } \\
\cline { 2 - 7 } & $\mathrm{N}^{\circ}$ & $\%$ & $\mathrm{~N}^{\circ}$ & $\%$ & $\mathrm{~N}^{\circ}$ & $\%$ \\
\hline $\begin{array}{l}\text { 7. En el DIAC, tiene bien esquematizado } \\
\text { cada parte curricular basada en los accesos } \\
\text { recursos didácticos. }\end{array}$ & $70 \%$ & 24 & $30 \%$ & 81 & $100 \%$ \\
$\begin{array}{l}\text { 8. En el DIAC, el entorno escolar tiene todos } \\
\text { los implementos necesarios para cada NEE. }\end{array}$ & & & & & & \\
$\begin{array}{l}\text { 9. En el DIAC, el entorno escolar es } 53 \\
\text { inclusivo con descripciones en el PCI }\end{array}$ & $65 \%$ & 28 & $35 \%$ & 81 & $100 \%$ \\
\end{tabular}

Fuente: Lista de cotejo aplicado a los docentes del Educación Básica Circuito 09D05C04 de Guayaquil.

Figura 3 Niveles y porcentajes del indicador factor del entorno escolar

\section{Factor del entorno escolar}

9. En el Documento Individual de Adaptación Curricular, el entorno escolar es inclusivo con...

8. En el Documento Individual de Adaptación Curricular, el entorno escolar tiene todos los...

7. En el Documento Individual de Adaptación Curricular, tiene bien esquematizado cada parte...

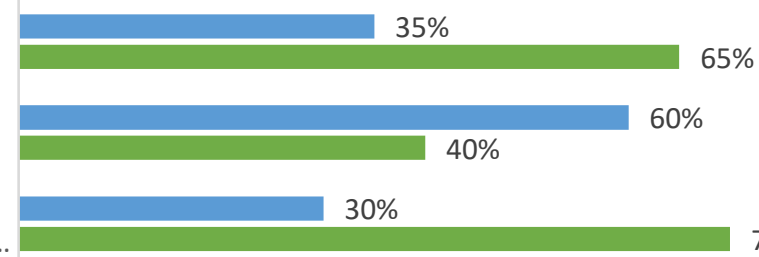

Fuente: Lista de cotejo aplicado a los docentes del Educación Básica Circuito 09D05C04 de Guayaquil. 
El factor del entorno escolar, se observa que el $70 \%$ no tuvo esquematizado cada parte del currículo basados en los accesos y recursos didácticos que requieren los estudiantes con NEE, es evidente entonces que, el $40 \%$ no contaron con implementos necesarios para los estudiantes, cabe agregar que, el $65 \%$ el entorno escolar no es inclusivo y no se encontró normado en el Proyecto Educativo Institucional. El entorno escolar requiere de inclusión educativa con esquemas de acceso y recursos, implementos necesarios para las NEE dentro del Proyecto Educativo Institucional.

OE2: Identificar los elementos que debe tener una guía de adaptaciones curriculares para docentes, que permita mejorar el rendimiento académico en los niños con NEE de Educación Básica de la ciudad de Guayaquil.

Tabla 5 Datos de la dimensión evaluación cuantitativa: Instrumentos de evaluación.

\begin{tabular}{|c|c|c|c|c|c|c|}
\hline \multirow[b]{2}{*}{ Instrumentos de evaluación } & \multicolumn{2}{|c|}{ NO } & \multicolumn{2}{|l|}{ SI } & \multicolumn{2}{|c|}{ Total } \\
\hline & $\mathrm{N}^{\circ}$ & $\%$ & $\mathrm{~N}^{\circ}$ & $\%$ & $\mathrm{~N}^{\circ}$ & $\%$ \\
\hline 10. Cuenta con & 52 & $64 \%$ & 29 & $36 \%$ & 81 & $100 \%$ \\
\hline $\begin{array}{l}\text { Planificación Curricular } \\
\text { Institucional con la } \\
\text { descripción de cada NEE }\end{array}$ & & & & & & \\
\hline $\begin{array}{l}\text { 11. Tiene el manual de } \\
\text { estrategias pedagógicas para } \\
\text { Atender la Necesidades } \\
\text { Educativas Especiales en la } \\
\text { Educación Regular. }\end{array}$ & 43 & $53 \%$ & 38 & $47 \%$ & 81 & $100 \%$ \\
\hline $\begin{array}{l}\text { 12. Tiene guía de } \\
\text { adaptaciones curriculares } \\
\text { para la educación inclusiva }\end{array}$ & 52 & $64 \%$ & 29 & $36 \%$ & 81 & $100 \%$ \\
\hline
\end{tabular}

Fuente: Lista de cotejo aplicado a los docentes del Educación Básica Circuito 09D05C04 de Guayaquil.

Figura 4 Niveles y porcentajes del indicador de instrumentos de evaluación

\section{Instrumentos de evaluación}

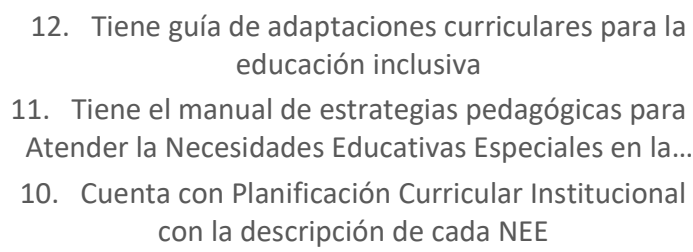

11. Tiene el manual de estrategias pedagógicas para Atender la Necesidades Educativas Especiales en la...

10. Cuenta con Planificación Curricular Institucional con la descripción de cada NEE

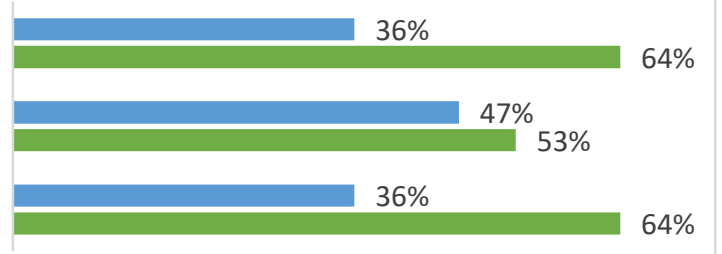

Fuente: Lista de cotejo aplicado a los docentes del Educación Básica Circuito 09D05C04 de Guayaquil.

La dimensión de evaluación cuantitativa describe el indicador de instrumentos de evaluación, donde el 64\% no cuenta con la planificación curricular institucional con la descripción de cada NEE que tiene en el centro educativo, en este mismo orden, el 53\% no le proporcionaron de manera digital el manual de estrategias pedagógicas para atender las necesidades educativas especiales en la educación regular, también, el 64\% tiene alguna guía de adaptaciones curriculares para la educación inclusiva. Esto 
implica que los docentes no tienen especificado que instrumentos de evaluación requieres para los estudiantes con NEE por la falta de guías o manuales que faciliten la labor educativa.

Tabla 6 Datos de la dimensión evaluación cuantitativa: Criterios de evaluación.

\begin{tabular}{|c|c|c|c|c|c|c|}
\hline \multirow[b]{2}{*}{ Criterios de evaluación } & \multicolumn{2}{|l|}{$\mathrm{NO}$} & \multicolumn{2}{|l|}{ SI } & \multicolumn{2}{|c|}{ Total } \\
\hline & $\mathrm{N}^{\circ}$ & $\%$ & $\mathrm{~N}^{\circ}$ & $\%$ & $\mathrm{~N}^{\circ}$ & $\%$ \\
\hline $\begin{array}{l}\text { 13. Dispone del registro de } \\
\text { desempeño a estudiantes con } \\
\text { adaptaciones curriculares en la }\end{array}$ & 52 & $64 \%$ & 29 & $36 \%$ & 81 & $100 \%$ \\
\hline $\begin{array}{l}\text { Planificación } \\
\text { Institucional. }\end{array}$ & & & & & & \\
\hline $\begin{array}{l}\text { 14. Estipula los criterios de } \\
\text { desempeño de los estudiantes con } \\
\text { NEE en la Planificación } \\
\text { Curricular Institucional. }\end{array}$ & 53 & $65 \%$ & 28 & $35 \%$ & 81 & $100 \%$ \\
\hline
\end{tabular}

Fuente: Lista de cotejo aplicado a los docentes del Educación Básica Circuito 09D05C04 de Guayaquil.

Figura 5 Niveles y porcentajes del indicador criterios de evaluación

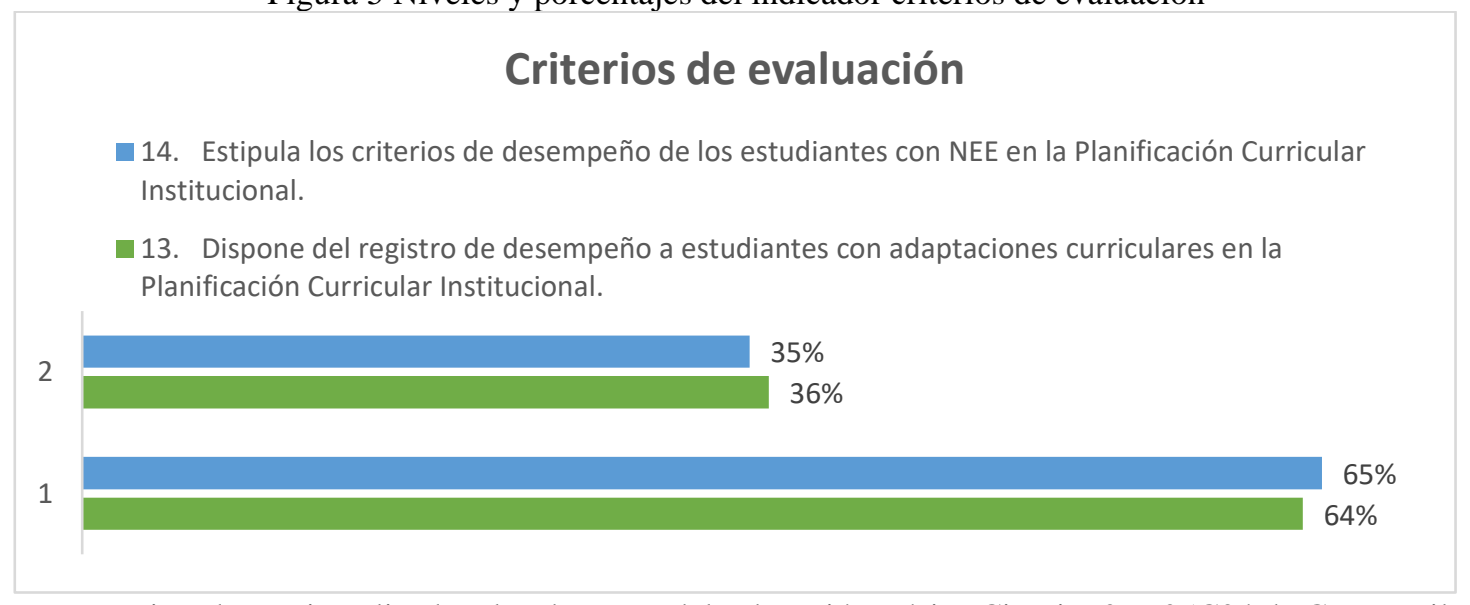

Fuente: Lista de cotejo aplicado a los docentes del Educación Básica Circuito 09D05C04 de Guayaquil.

Los resultados en la dimensión de evaluación cuantitativa de los criterios de evaluación el 64\% no disponían de registros de desempeño a estudiantes con adaptaciones curriculares en la Planificación Curricular Institucional, cabe agregar, que el $65 \%$ tampoco estipuló los criterios de desempeño de los estudiantes con NEE en dicha planificación. Los criterios de evaluación de los estudiantes con necesidades educativas especiales con o sin discapacidad no son especificados en el documento oficial de los establecimientos educativos del Distrito 09D05 de la ciudad de Guayaquil.

OE3: Describir las características que tienen las estrategias diversificadas y específicas que utilizan los docentes para el rendimiento académico de los estudiantes con NEE. 
Tabla 7 Datos de la dimensión estrategias para la atención de discapacidad: Estrategias específicas para la discapacidad intelectual, auditiva y visual.

\begin{tabular}{|c|c|c|c|c|c|c|}
\hline \multicolumn{3}{|c|}{ Estrategias específicas para $\mathrm{NO}$} & \multicolumn{2}{|l|}{ SI } & \multicolumn{2}{|c|}{ Total } \\
\hline la discapacidad & $\mathrm{N}^{\circ}$ & $\%$ & $\mathrm{~N}^{\circ}$ & $\%$ & $\mathrm{~N}^{\circ}$ & $\%$ \\
\hline $\begin{array}{l}\text { 15. La docente cuenta con } \\
\text { estrategias para el acceso a la } \\
\text { I.E que requiere el estudiante } \\
\text { con NEE. }\end{array}$ & 43 & $53 \%$ & 38 & $47 \%$ & 81 & $100 \%$ \\
\hline $\begin{array}{l}\text { 16. Presenta estrategias } \\
\text { acordes a los problemas de } \\
\text { aprendizaje que tienen los } \\
\text { estudiantes con NEE. }\end{array}$ & 52 & $64 \%$ & 29 & $36 \%$ & 81 & $100 \%$ \\
\hline $\begin{array}{l}17 . \quad \text { Considera las } \\
\text { adaptaciones curriculares de } \\
\text { acuerdo a las necesidades de } \\
\text { los estudiantes con NEE. }\end{array}$ & 61 & $75 \%$ & 20 & $25 \%$ & 81 & $100 \%$ \\
\hline
\end{tabular}

Figura 6 Niveles y porcentajes del indicador estrategias específicas para la discapacidad

\section{Estrategias específicas para la discapacidad}

17. Considera las adaptaciones curriculares de acuerdo a las necesidades de los estudiantes con NEE.

16. Presenta estrategias acordes a los problemas de aprendizaje que tienen los estudiantes con NEE.

15. La docente cuenta con estrategias para el acceso a la I.E que requiere el estudiante con NEE.

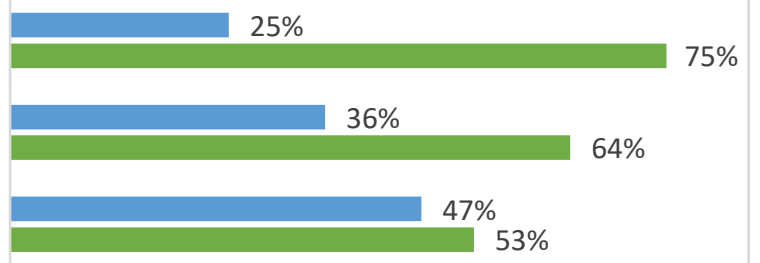

Fuente: Lista de cotejo aplicado a los docentes del Educación Básica Circuito 09D05C04 de Guayaquil.

Los datos que se representan en la tabla 6 comprenden el 53\% de los docentes que no contaron con estrategias para el acceso a la IE que requiere un estudiante con NEE; de manera similar en el 64\% no se observó que tenga estrategias acordes a los problemas de aprendizaje que tienen los estudiantes, en el mismo sentido el $75 \%$ no consideró las adaptaciones curriculares de acuerdo a las necesidades de los estudiantes. Es decir, que los docentes poco implementan estrategias para la discapacidad intelectual, auditiva y visual.

Tabla 8 Datos de la dimensión estrategias para la atención de discapacidad: Estrategias específicas para la discapacidad psicosocial. Síndrome de Asperger, trastorno por déficit de atención o trastornos específicos de aprendizaje

\begin{tabular}{|c|c|c|c|c|c|c|}
\hline \multirow[t]{2}{*}{ específicas } & \multicolumn{2}{|l|}{ la NO } & \multicolumn{2}{|l|}{ SI } & \multicolumn{2}{|c|}{ Total } \\
\hline & $\mathrm{N}^{\circ}$ & $\%$ & $\mathrm{~N}^{\circ}$ & $\%$ & $\mathrm{~N}^{\circ}$ & $\%$ \\
\hline $\begin{array}{l}\text { 18. La docente cuenta con estrategias para } \\
\text { el acceso a la I.E a estudiantes con NEE. }\end{array}$ & 44 & $54 \%$ & 37 & $46 \%$ & 81 & $100 \%$ \\
\hline $\begin{array}{l}\text { 19. Presenta estrategias acordes a los } \\
\text { problemas de aprendizaje que tienen los } \\
\text { estudiantes con NEE. }\end{array}$ & s 40 & $49 \%$ & 41 & $51 \%$ & 81 & $100 \%$ \\
\hline $\begin{array}{l}\text { 20. Considera las adaptaciones } \\
\text { curriculares de acuerdo a las necesidades } \\
\text { de los estudiantes con NEE. }\end{array}$ & es 51 & $63 \%$ & 30 & $37 \%$ & 81 & $100 \%$ \\
\hline
\end{tabular}


Figura 7 Niveles y porcentajes del indicador estrategias específicas para la discapacidad

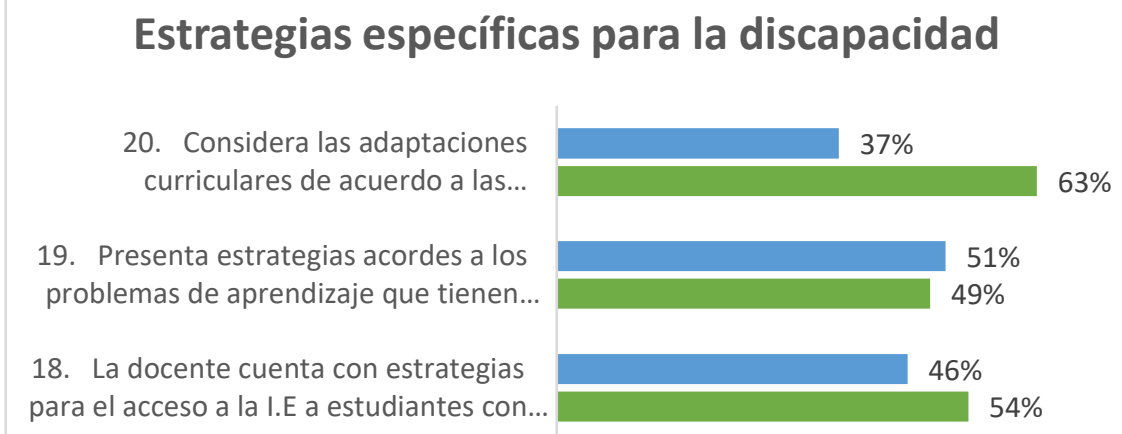

Fuente: Lista de cotejo aplicado a los docentes del Educación Básica Circuito 09D05C04 de Guayaquil.

En la misma dimensión, pero con otras estrategias específicas, el 54\% de los docentes no contó con estrategias de acceso a la IE para niños con discapacidad psicosocial, síndrome de Asperger, trastorno por déficit de atención o trastornos específicos de aprendizaje, de la misma manera, el $49 \%$ no presenta estrategias acordes a los problemas de aprendizaje y así mismo, el 63\% no ha considerado la adaptación curricular. De tal manera, los docentes no han empleado estrategias específicas para estudiantes con discapacidad psicosocial, síndrome de Asperger, trastorno por déficit de atención o trastornos específicos de aprendizaje

Tabla 9 Datos de la dimensión estrategias para la atención de discapacidad: Estrategias diversificadas.

\begin{tabular}{|c|c|c|c|c|c|c|}
\hline \multirow[b]{2}{*}{ Estrategias diversificadas } & \multicolumn{2}{|l|}{$\mathrm{NO}$} & \multicolumn{2}{|l|}{ SI } & \multicolumn{2}{|c|}{ Total } \\
\hline & $\mathrm{N}^{\circ}$ & $\%$ & $\mathrm{~N}^{\circ}$ & $\%$ & $\mathrm{~N}^{\circ}$ & $\%$ \\
\hline $\begin{array}{l}\text { 21. El docente cuenta con estrategias para } \\
\text { el acceso a la I.E de estudiantes con NEE. }\end{array}$ & 58 & $72 \%$ & 23 & $28 \%$ & 81 & $100 \%$ \\
\hline $\begin{array}{l}\text { 22. Presenta estrategias acordes a los } \\
\text { problemas de aprendizaje que tienen los } \\
\text { estudiantes con NEE. }\end{array}$ & 61 & $75 \%$ & 20 & $25 \%$ & 81 & $100 \%$ \\
\hline $\begin{array}{l}\text { 23. Considera las adaptaciones } \\
\text { curriculares de acuerdo a las necesidades } \\
\text { de los estudiantes con NEE. }\end{array}$ & 62 & $77 \%$ & 19 & $23 \%$ & 81 & $100 \%$ \\
\hline
\end{tabular}

Figura 8 Elementos y porcentajes del indicador estrategias diversificadas

\section{Estrategias diversificadas}

23. Considera las adaptaciones curriculares de acuerdo a las necesidades de los estudiantes con NEE.

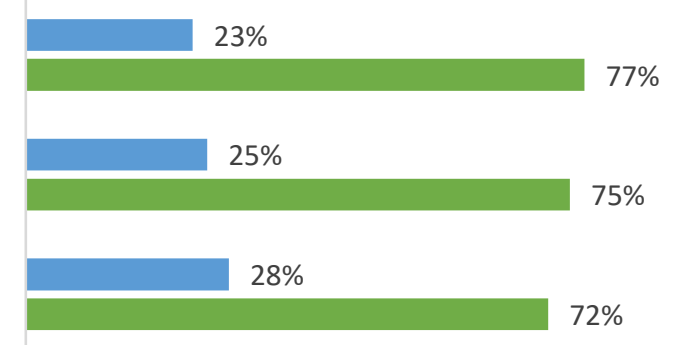

Fuente: Lista de cotejo aplicado a los docentes del Educación Básica Circuito 09D05C04 de Guayaquil. 
Por último, en la dimensión de estrategias para la atención de discapacidad en el indicador de estrategias diversificadas, el 72\% de los educadores no contó con estrategias de acceso, seguidamente, el 75\% no especificó los problemas de aprendizaje y al mismo tiempo, el 77\% no consideró las adaptaciones curriculares basadas en la necesidad del estudiante. Se concluye que los docentes no emplean estrategias diversificadas para atender las necesidades educativas especiales.

OE4: Identificar los elementos que aportan a la propuesta para reducir las causas del rendimiento académico en niños con NEE de Educación Básica Circuito 09D05C04 de Guayaquil, 2021.

Tabla 10 Elementos destacados para la elaboración de la propuesta

\begin{tabular}{|c|c|c|}
\hline Dimensión & Tabla & Elemento \\
\hline \multirow{3}{*}{ Factores del rendimiento escolar } & $\mathrm{N}^{\circ} 1$ & $\begin{array}{l}\text { Escases de responsabilidad de las familias en la educación } \\
\text { del estudiante con NEE. }\end{array}$ \\
\hline & $\mathrm{N}^{\circ} 2$ & $\begin{array}{l}\text { Pocas adaptaciones de las habilidades sociales con } \\
\text { especialistas internos y externos. }\end{array}$ \\
\hline & $\mathrm{N}^{\circ} 3$ & $\begin{array}{l}\text { Insuficiente Inclusión educativa en documentos legales } \\
\text { del plantel. }\end{array}$ \\
\hline \multirow{2}{*}{ Evaluación cuantitativa } & $\mathrm{N}^{\circ} 4$ & $\begin{array}{l}\text { Falta de guía de adaptaciones curriculares con la } \\
\text { descripción de cada NEE. }\end{array}$ \\
\hline & $\mathrm{N}^{\circ} 5$ & $\begin{array}{l}\text { Poco registro de desempeño y criterios de evaluación de } \\
\text { estudiantes con NEE. }\end{array}$ \\
\hline \multirow{3}{*}{$\begin{array}{l}\text { Estrategias para la atención de } \\
\text { discapacidad }\end{array}$} & $\mathrm{N}^{\circ} 6$ & $\begin{array}{l}\text { Pocas estrategias acordes a los problemas y necesidades } \\
\text { de los estudiantes con NEE discapacidad intelectual, } \\
\text { auditiva y visual. }\end{array}$ \\
\hline & $\mathrm{N}^{\circ} 7$ & $\begin{array}{l}\text { Los docentes no emplean adaptaciones curriculares de } \\
\text { acuerdo a las necesidades de los estudiantes la } \\
\text { discapacidad psicosocial. Síndrome de Asperger, } \\
\text { trastorno por déficit de atención o trastornos específicos } \\
\text { de aprendizaje. }\end{array}$ \\
\hline & $\mathrm{N}^{\circ} 8$ & $\begin{array}{l}\text { Los docentes no emplean estrategias diversificadas para } \\
\text { atender las necesidades educativas especiales. }\end{array}$ \\
\hline
\end{tabular}

Fuente: Resultados del análisis e interpretación de la lista de cotejo.

En la tabla 10 se registran los resultados obtenidos en el análisis de cada dimensión, describiendo los elementos indispensables para la propuesta de las adaptaciones curriculares. En la dimensión factores del rendimiento académico la escasa responsabilidad de las familias, como el desarrollo de las habilidades sociales. En la dimensión de evaluación cuantitativa, el establecimiento educativo requiere del Documento Individual de Adaptación Curricular requiere de los documentos legales que respalden la NEE, la respectiva guía de adaptación curricular con las descripciones específicas, las planificaciones que describan los desempeños y criterios de evaluación, en la dimensión de estrategias para la atención de discapacidad la implementación de las adaptaciones para los estudiantes con NEE con o sin discapacidad.

\section{DISCUSIÓN}

En el objetivo específico 1 consiste en caracterizar las adaptaciones curriculares de estudiantes con NEE que realizan los docentes de las aulas inclusivas a los docentes del Educación Básica Circuito 
09D05C04 de Guayaquil; lo cual, de los 81 participantes que formaron parte de la técnica de observación con el instrumento lista de cotejo, en el indicador "factor del entorno familiar" el 58\% no cuentan con el documento individual de adaptación curricular y las respectivas descripciones de los integrantes del hogar. En ese mismo sentido, el $54 \%$ de los educadores no ha detallado la relación de las familias en la discapacidad del estudiante con algún problema de aprendizaje o discapacidad de algún familiar, mientras que el $75 \%$ no detalla las responsabilidades que tienen los familiares en la educación que recibe el estudiante, lo cual ha permitido identificar que en las características de las adaptaciones curriculares de estudiantes con NEE no se evidencia la responsabilidad de los familiares, en cuanto al conocimiento que requieren los estudiantes para aprender las destrezas con criterio de desempeño ni la responsabilidad que deben asumir los docentes (Tabla 1).

De la misma manera, el indicador factor del entorno social que tienen los estudiantes con NEE, el porcentaje elevado, por ejemplo, el $67 \%$ no cuentan con las respectivas adaptaciones curriculares de las habilidades sociales que necesitan los infantes; del mismo modo, el $70 \%$ no han planteado actividades que sean interactivas con el resto del salón de clases; también, el 59\% no ha detallado la intervención de los especialistas internos y externos que deben contar los estudiantes con NEE. Es notable que se han empleado pocas adaptaciones curriculares sobre las habilidades sociales de los estudiantes, uno de los factores que no permiten la interrelación entre todos los infantes. (Tabla 2)

Por último, el factor del entorno escolar dos de las tres interrogantes son destacadas en porcentajes, debido a que el $70 \%$ de los encuestados no tienen bien esquematizado cada parte curricular en el tipo de acceso y recursos didácticos que deben contar para realizar las clases, del mismo sentido, el 65\% no refleja que tengan el entorno inclusivo en las descripciones del Proyecto Curricular Institucional esto quieres decir, que existe insuficiente inclusión educativa en documentos legales del plantel. (Tabla 3).

Los resultados antes presentados, concuerda con los obtenidos en la investigación de Sabando (2016) en su trabajo doctoral titulado "inclusión educativa y rendimiento escolar" en la discusión indica el 54\% que se debe promover el diseño de evaluaciones a los centros educativos para que garanticen la inclusión educativa, el $62 \%$ precisa los aprendizajes de los estudiantes con estrategias acordes a sus potencialidades, adaptando según la necesidad, de la misma manera, el 35\% impulsar la co-docencia que favorezca a los niños con NEE para generar aulas con espacios, tiempos, recursos que permitan enseñar de manera significativa. De la misma manera, se relaciona con el antecedente de Viloria (2016) titulado "La integración escolar del alumnado con necesidades educativas especiales en el Municipio Caroní de Venezuela", quién en la discusión, afirma que el 65\% de los casos de integración escolar está vinculado al ámbito de la sociedad como parte fundamental para el aprendizaje de los niños con NEE, además, el $57 \%$ de los padres de familia no describen las necesidades educativas de sus representados ocasionando 
un retraso en el aprendizaje, según las diferentes adaptaciones curriculares que pueden recibir si lo mencionan desde el inicio escolar.

Los resultados antes presentados se relacionan con la teoría de (Chong, 2017), para quien los tres factores, tanto el familiar, social y escolar son habilidades apropiadas para el desarrollo socioeconómico, la capacidad de responsabilidad que tienen los familiares, para potenciar la mejora de calidad educativa en la inclusión en los espacios escolares. Los planes de estudios con las adaptaciones apropiadas logran un rendimiento escolar viable para la vida cotidiana.

El objetivo específico 2 consiste en Identificar los elementos que debe tener una guía de adaptaciones curriculares para docentes, que permita mejorar el rendimiento académico en los niños con NEE de Educación Básica de la ciudad de Guayaquil. El 64\% de los documentos observados no cuentan con las planificaciones curriculares con la descripción del estudiante con NEE, el 53\% no cuenta con el manual de estrategias pedagógicas, el 64\% no tienen la guía de adaptaciones curriculares (Tabla $\mathrm{N}^{\circ}$. 5).

En la dimensión de evaluación cuantitativa indicador criterio de evaluación el 64\% no registran el desempeño de los escolares, en cambio, el 65\% no estipula los criterios de desempeño de los estudiantes con NEE en la planificación curricular anual (Tabla Nº. 6). Los resultados analizados de Encalada (2019) en el diagnóstico realizado a los docentes evidencia el 100\% no registran el tipo de evaluaciones deben realizar a los estudiantes con NEE, además, el 93\% del mismo sentido, las adecuaciones curriculares no consideran los criterios de evaluación.

Relacionando lo antes expuesto con la teoría de (Azorín, 2017) los documentos institucionales que todo educador debe contar, por ejemplo, el DIAC, los manuales y guías de las adaptaciones curriculares y sobre todo la planificación curricular anual, donde tenga estipulado que tipo de instrumentos y criterios de evaluación se emplea para los estudiantes que tienen NEE, como una práctica inclusiva basadas en estrategias específicas o diversificadas que requiera la discapacidad ya sea permanente o transitoria.

El objetivo específico 3 Describir las características que tienen las estrategias diversificadas y específicas que utilizan los docentes para el rendimiento académico de los estudiantes con NEE. El 53\% de los registros observados los docentes no cuentan con estrategias de acceso para los estudiantes con NEE, el 64\% tampoco presente estrategias acordes a los problemas de aprendizajes y el $75 \%$ las adaptaciones curriculares no están acuerdo a las NEE (Tabla $\mathrm{N}^{\circ}$ 7). Del mismo sentido, el 54\% de los registros no cuentan con estrategias de atención a la discapacidad psicosocial. Síndrome de Asperger, trastorno por déficit de atención o trastornos específicos de aprendizaje, el $49 \%$ tampoco cuenta con estrategias acordes a los problemas de aprendizajes, el 63\% no ha considerados estas adaptaciones en ninguno de sus registros institucionales (Tabla 8). En relación a las estrategias diversificadas el $72 \%$ de los registros observados los docentes no cuentan con estrategias para el acceso, el $75 \%$ tampoco presenta las estrategias de aprendizaje y el $77 \%$ no considera las adaptaciones para este tipo de NEE (Tabla 9). 
Los resultados obtenidos concuerdan con los de Chávez (2016) pues refleja en su estudio que el $56 \%$ los docentes carecen de conocimiento de los tipos de adaptaciones curriculares que se deben emplear para la inclusión educativa, además, el $46 \%$ de los educadores aprecian que la inclusión educativa es una realidad latente en las aulas escolares. Ambos aportes sustentan la teoría de varios autores que describen las estrategias implementadas en diferentes contextos, por ejemplo, de Cornejo (2017) permitiendo conocer que las estrategias específicas para la discapacidad auditiva son las habilidades lingüísticas, a través del lenguaje de señas. Para Barreiro (2018) las estrategias específicas de la discapacidad física son las de acceso al establecimiento educativo. En cambio, Paredo, (2016) para la discapacidad intelectual son los apoyos externos, es decir, los especialistas como psicólogos, terapistas, psicopedagógicos. Del mismo sentido, Figueroa (2017) las estrategias son la motivación, clima de confianza. Las adaptaciones curriculares desarrollan las habilidades cognitivas y físicas de los estudiantes según su nivel de dificultades (Echeverría, 2017).

En el objetivo específico 4 Identificar los elementos que aportan a la propuesta para reducir las causas del rendimiento académico en niños con NEE de Educación Básica Circuito 09D05C04 de Guayaquil, 2021, se basa en el sustento de los elementos destacados de los resultados obtenidos en la observación con el instrumento de la lista de cotejo, donde se describen tres dimensiones que arrojan más del $50 \%$ de registros no presentados por parte del docente, el primer factor denominado rendimiento escolar, presentando poca responsabilidad que tienen las familias con las habilidades sociales de los niños con NEE, la dimensión de evaluación cuantitativa sin contar con documentos legales como el DIAC, la guía y manual para seleccionar el adecuado criterio de evaluación, por último, la dimensión de estrategias para la atención de la discapacidad, ya sea permanente o transitoria con pocas estrategias para las discapacidades: intelectual, auditiva, visual, psicosocial, Síndrome de Asperger, trastorno por déficit de atención o trastornos específicos de aprendizaje.

\section{CONCLUSIONES}

Los directivos del Distrito 09D05C04 poco registran en los documentos legales de los centros educativos, las responsabilidades que tienen las familias de los estudiantes con NEE para desarrollar las habilidades sociales, las actividades interactivas, las adaptaciones con la ayuda externa de expertos en el tema.

Los centros educativos carecen de los elementos que deben tener una guía, por ejemplo, Documento Individual de Adaptación Curricular, la guía y manual de adaptaciones curriculares, insumos que permiten registrar el desempeño y los criterios de evaluación adecuados en base a las estrategias específicas y diversificadas para cada NEE. 
Los educadores no cuentan con estrategias específicas y diversificadas para los estudiantes con NEE, desconociendo este tipo de actividades que pueden implementar en las adaptaciones curriculares según las discapacidades o problemas de aprendizaje.

Los índices mayores del 50\% han determinado que los elementos que aportan a una propuesta para mejorar el rendimiento académico de los niños con NEE son: factores del rendimiento escolar, evaluación cuantitativa y las estrategias para la atención a la discapacidad.

Los planteles del Distrito 09D05C04 deben contar con una guía de adaptaciones curriculares para mejorar el rendimiento académico de los estudiantes con NEE. 


\section{REFERENCIAS}

Carrión, M. (2019). Inclusión educativa de estudiantes con necesidades educativas especiales permanentes en la Universidad Técnica de Machala-Ecuador, 2017. Lima-Perú: Universidad Nacional Mayor de San Marcos. Obtenido de http://scielo.sld.cu/scielo.php?script=sci_arttext\&pid=S199086442019000300195

Chávez, M. (2016). Adaptaciones curriculares e inclusión escolar con discapacidad intelectual a partir de la percepción de los docentes de primaria, Ica-2016. Perú: Universidad César Vallejo. Obtenido de https://repositorio.ucv.edu.pe/handle/20.500.12692/18897

Constitución de la República del Ecuador, C. (2008). Constitución de la República del Ecuador 2008. Quito: Lexis.

Guterres, A. (2019). Estamos muy lejos del progreso hacia el desarrollo sostenible. UNESCO, 1-19. Obtenido de https://www.un.org/sustainabledevelopment/es/2019/11/estamos-muy-lejos-del-progresohacia-el-desarrollo-sostenible/

López, R. (2018). Un estudio sobre la situación de la educación inclusiva en centros educativos desde la percepción de la comunidad educativa. Cádiz-España: Universidad de Cadiz.

Martínez, J. R. (2020). Estrategias pedagógicas inclusivas y su aporte en el aprendizaje de los estudiantes con necesidades educativas especiales. Revista de Investigación e innovación Magazine de las Ciencias, 5( $\mathrm{N}^{\circ}$ CISE 2020), $\quad 354-369 . \quad$ Obtenido de https://www.google.com/search?q=estrategias+especificas+de+la+discapacidad+pdf\&rlz=1C1SQJL_es EC878EC878\&ei=qQ2fYf--

FsuZwbkP5PSK2Ak\&start=30\&sa=N\&ved=2ahUKEwi_t9ax07L0AhXLTDABHWS6Aps4FBDw0wN $6 \mathrm{BAgBEE} 0 \&$ biw $=1366 \& \mathrm{bih}=625 \& \mathrm{dpr}=1 \#$

Meléndez, R. (2018). Educación inclusiva y discapacidad en Costa Rica: una perspectica desde las politicas publicas. Actualidades Investigativas en Educación. Actualidades Investigativas en Educación, vol. 18(núm. 2), 1-27. Obtenido de http://www.redalyc.org/articulo.oa?id=44758022019

Moliner, O. R. (2020). Procesos de movilización del conocimiento sobre educación inclusiva en las escuelas suizas. ¿Qué papel juegan los investigadores/ formadores que asesoran los procesos de mejora escolar? Estudios Sobre Educación, vol. 38, 169-189. Obtenido de https://revistas.unav.edu/index.php/estudios-sobre-educacion/article/view/38673

Ortigosa, I. (2016). Adaptación curricular para alumnos con discapacidad visual en el aula de matemáticas. Almería-España: Universidad de Almería. Obtenido de http://repositorio.ual.es/handle/10835/6066

Parts, J. (1 de 03 de 2020). Universidad de Barcelona. Obtenido de http://www.ub.edu/histodidactica/images/documentos/pdf/Mario\%20Bunge.pdf

Plancarte, P. (2017). Inclusión educativa y cultura inclusiva. Revista Nacional e Internacional de Educación Inclusiva, vol. 10(núm. 2), 213-226. Obtenido de https://dialnet.unirioja.es/servlet/articulo?codigo $=6545223$ 
Rátiva, L. Q. (2016). Proyecto de aula potenciador de la dimensión comunicativa desde la zona de desarrollo próximo. Colombia: Universidad Libre de Colombia.

Romero, J. L. (2003). Dificultades en el Aprendizaje: Unificación de Criterios Dianósticos. México: Junta de Andalucía. Obtenido de https://www.uma.es/media/files/LIBRO_I.pdf

Sabando, D. (2016). Inclusión educativa y rendimiento académico. Barcelona-España: universitat de Barcelona. Obtenido de https://www.tesisenred.net/bitstream/handle/10803/402901/DSSR_TESIS.pdf?sequence=3\&isAllowed= $\mathrm{y}$

Tolentino, H. (2020). Habilidades sociales y estrategias didácticas para la formación del liderazgo desde la educación básica. Revista Educación, 44(2), 1-15. Obtenido de https://www.redalyc.org/jatsRepo/440/44062184036/44062184036.pdf 